\title{
Influência de fatores afetivos, antropométricos e sociodemográficos sobre o comportamento alimentar em jovens atletas
}

\author{
Influence of affective, anthropometric and sociodemographic \\ factors on eating behavior in young athletes \\ Leonardo de Sousa Fortes', Flávio Garcia de Oliveira', Maria Elisa Caputo Ferreira²
}

\section{RESUMO}

Objetivo: $\bigcirc$ objetivo do estudo foi analisar a influência de diversos fatores sobre o comportamento alimentar inadequado (CAl) em jovens atletas femininas. Métodos: Participaram 116 esportistas. Avaliou-se o CAl mediante aplicação do Eating Attitudes Test (EAT-26). A insatisfação corporal e o comprometimento psicológico ao exercício foram avaliados pelo Body Shape Questionnaire e pelo Commitment Exercise Scale, respectivamente. O nível econômico foi obtido pelo "Critério de Classificação Econômica Brasil". Aferiram-se peso e estatura para calcular o índice de massa corporal (IMC) e dobras cutâneas para estimar o percentual de gordura. Conduziu-se regressão linear múltipla utilizando o software SPSS 17.0 e adotando nível de significância de 5\%. Resultado: A insatisfação corporal, seguida do percentual de gordura, foram as únicas variáveis que modularam as pontuações do EAT-26. Contudo, o mo-

\section{Palavras-chave}

Comportamento alimentar, imagem corporal, atletas. delo de regressão linear múltipla explicou um terço da variância do CAI nessas esportistas. Conclusão: Concluiu-se que o CAl de atletas femininas foi influenciado basicamente pela insatisfação corporal.

\section{ABSTRACT}

Objective: The aim of this study was to analyze the influence of various factors on the inadequate eating behavior (IEB) in young female athletes. Methods: Participants 116 athletes. The IEB was evaluated by applying IEB of the Eating Attitudes Test (EAT-26). The body dissatisfaction and psychological commitment to exercise were assessed by the Body Shape Questionnaire and Commitment Exercise Scale, respectively. The economic level was obtained by the "Classification Criteria Economic Brazil". Body weight and height to calculate the index of mass (BMI) and skinfold thickness to estimate the percentage of fat. We carried out multiple linear regression using the SPSS 17.0 software and adopting a significance level of 5\%. Result: The body dissatisfaction, followed by the percentage of fat were the only variables that modulate the EAT-26 scores. However, the multiple linear regression model explained 1/3 of the variance of these IEB sportsmen. Conclusion: It was concluded that the IEB was influenced female athletes primarily by body dissatisfaction.

Eating behavior, body

image, athletes.

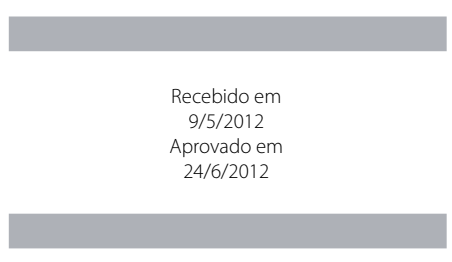

1 Universidade Federal de Juiz de Fora (UFJF), Conselho Nacional de Desenvolvimento Científico e Tecnológico (CNPq), Grupo de Pesquisa "Corpo e Diversidade Humana".

2 Universidade de São Paulo (USP); UFJF, Departamento de Fundamentos da Educação Física, CNPq, Grupo de Pesquisa "Corpo e Diversidade Humana". 


\section{INTRODUÇÃO}

Os transtornos alimentares (TA) são síndromes psicológicas caracterizadas por controle patológico do peso corporal, distúrbios da percepção do formato corporal e comportamento alimentar gravemente perturbado ${ }^{1,2}$. É comum identificar em pacientes com TA a utilização de medicamentos (laxantes e diuréticos) para emagrecimento, autoindução de vômitos, prática extenuante de exercício físico e restrição alimentar $^{3}$. No entanto, muitos indivíduos sem diagnóstico clínico de TA podem apresentar tais comportamentos, podendo ser considerados com riscos para o desenvolvimento dessas síndromes $^{4-6}$, como é o caso dos atletas ${ }^{7-9}$, principalmente os do sexo feminino ${ }^{10,11}$.

Existem vários fatores que podem influenciar o desenvolvimento do comportamento alimentar inadequado. Segundo Conti et al. ${ }^{12}$, a insatisfação corporal é o principal deles. É denominada como a preocupação com o peso e a aparência física ${ }^{13,14}$. Estudos têm apontado que a inadequação alimentar é fruto da insatisfação corporal14,15.

Além da insatisfação com o corpo, autores têm apontado que a inadequação morfológica pode levar à alimentação desordenada em jovens ${ }^{14,16}$, ou seja, altos valores de índice de massa corporal (IMC) e percentual de gordura têm sido associados aos hábitos alimentares anormais ${ }^{13,17}$. Ademais, parece que o elevado grau de comprometimento psicológico ao exercício também pode influenciar negativamente o comportamento alimentar. Modolo et al. ${ }^{18}$ salientam que a dependência psicológica ao exercício físico pode ser consequência da depreciação do próprio corpo, podendo, então, prejudicar os hábitos alimentares.

O nível econômico e a etnia parecem ser agentes influenciadores para a instalação do comportamento alimentar de risco para TA. Pesquisadores afirmam que o comportamento alimentar inadequado é mais presente nos estratos mais altos da sociedade ${ }^{19}$, assim como é mais prevalente em sujeitos de etnia branca ${ }^{20}$.

Em atletas, o risco de desenvolver hábitos alimentares anormais parece ser maior. Krentz e Warschburger ${ }^{10}$ e Schaal et al. ${ }^{2}$ argumentam que o âmbito esportivo possui agentes potencializados para tais riscos. O nível competitivo é considerado um deles. Segundo Denoma et al.7, a inadequação alimentar é mais acentuada em jovens atletas que competem em níveis elevados, por causa da pressão imposta por treinadores, pais e patrocinadores.

Estudos têm apresentado grande prevalência de comportamentos alimentares inadequados em atletas $27,9,10,17$. Parece que o sexo feminino é mais acometido por esses comportamentos ${ }^{5,21}$. No entanto, nenhum estudo foi identificado até o momento averiguando a influência que fatores afetivos (insatisfação corporal), antropométricos (IMC e percentual de gordura) e sociodemográficos (etnia e nível competitivo) podem exercer sobre o comportamento alimentar inadequado em jovens atletas. Diante do pressuposto, o objetivo do presente estudo foi analisar a influência de diversos fatores sobre o comportamento alimentar inadequado em jovens atletas femininas.

\section{MÉTODOS}

Trata-se de um estudo transversal realizado nas cidades do Rio de Janeiro/RJ e Juiz de Fora/MG com jovens atletas do sexo feminino. Participaram 131 atletas adolescentes, pertencentes a cinco clubes (dois do Rio de Janeiro e três de Juiz de Fora), com idades entre 10 e 19 anos, participantes de competições e praticantes de diversas modalidades esportivas. No entanto, 15 jovens foram excluídas da pesquisa por não responderem aos questionários em sua totalidade ou por não participarem das aferições antropométricas, chegando a uma amostra de 116 atletas dos seguintes esportes: natação $(n=22)$, handebol $(n=20)$, basquetebol $(n=16)$, voleibol $(n=16)$, taekwondo $(n=5)$, ginástica artística $(n=$ 14), nado sincronizado $(n=18)$ e saltos ornamentais $(n=5)$. A amostra foi selecionada segundo alguns critérios: (1) ter disponibilidade para responder aos questionários e realizar aferições antropométricas; (2) ter idade entre 10 e 19 anos; (3) praticar sistematicamente sua respectiva modalidade esportiva por pelo menos três vezes na semana, com duração mínima de 1 hora por sessão; e (4) estar participando, no momento da coleta, de torneio de nível regional ou mais importante. Para seleção das participantes, adotou-se a técnica de amostragem casal simples descrita por Perini et al. ${ }^{5}$.

O projeto de pesquisa foi aprovado pelo Comitê de Ética e Pesquisa em Seres Humanos da Universidade Federal de Juiz de Fora, recebendo Parecer n 232/2010 (2149.209.2010), segundo a Resolução no 196/96, do Conselho Nacional de Saúde.

\section{Anamnese}

\section{Comportamento alimentar inadequado}

Avaliou-se mediante aplicação do questionário Eating Attitudes Test (EAT-26). O EAT-26 é composto por 26 itens em escala do tipo Likert que variam de 0 a 3 pontos (sempre $=$ 3 , muitas vezes $=2$, frequentemente $=1$, poucas vezes $=$ 0 , quase nunca $=0$ e nunca $=0$ ), com exceção da questão 25 , cuja pontuação é invertida (sempre $=0$, muitas vezes $=$ 0 , frequentemente $=0$, poucas vezes $=1$, quase nunca $=2$ e nunca $=3$ ). Essas questões estão distribuídas em três subescalas, a saber: a) dieta - diz respeito à recusa patológica a alimentos com alto teor calórico e preocupação com a aparência; b) bulimia e preocupação com os alimentos refere-se a episódios de compulsão alimentar, seguidos por comportamentos purgativos para perda/controle de peso corporal; e c) autocontrole oral - reflete o autocontrole em 
relação à comida e avalia forças ambientais e sociais estimulantes à ingestão alimentar. Escore igual ou maior que 20 representa indivíduos com comportamento alimentar de risco para TA. A versão utilizada no presente estudo foi validada por Bighetti et al. ${ }^{22}$ para jovens brasileiras do sexo feminino, que apresentou consistência interna de 0,82. Para a presente amostra, calculou-se o alfa de Cronbach, obtendo-se valor de 0,89 .

\section{Insatisfação corporal}

Avaliou-se por intermédio do Body Shape Questionnaire (BSQ). Trata-se de um teste de autopreenchimento que busca avaliar a frequência de preocupação com o peso e a aparência física. O BSQ é composto por 34 itens com formato de escala do tipo Likert variando de 0 a 6 (nunca $=0$ e sempre $=$ 6). A pontuação final é resultado da soma do escore de cada questão. Esse questionário classifica níveis a respeito da insatisfação corporal do sujeito. Quanto maior o escore, maior é a insatisfação com o corpo. A versão utilizada no presente estudo foi validada para adolescentes brasileiros por Conti et al. ${ }^{23}$, e sua análise de consistência interna revelou um alfa de 0,96 para ambos os sexos e significativo coeficiente de correlação entre os escores do teste-reteste, variando de 0,89 a 0,91 para meninas e meninos, respectivamente. Calculou-se o alfa de Cronbach para a amostra da presente pesquisa, encontrando-se valor de 0,91.

\section{Comprometimento psicológico ao exercício}

Para determinar o grau de comprometimento psicológico que um indivíduo possa ter com o hábito de se exercitar, aplicou-se a Commitment Exercise Scale (CES). O instrumento foi traduzido, adaptado e validado para a língua portuguesa por Teixeira et al. ${ }^{24}$, que apresentou alfa de Cronbach de 0,79. A CES avalia o grau com que sensações de bem-estar são moduladas pelo exercício, a manutenção do exercício em condições adversas e o grau de interferência que a atividade física tem em compromissos sociais do indivíduo. Trata-se de uma escala analógica visual, composta por oito questões que variam de 0 a $155 \mathrm{~mm}$ e, portanto, com uma pontuação máxima de 1.240 mm. Calculou-se a consistência interna para a presente amostra, identificando-se alfa de Cronbach de 0,85 .

\section{Antropometria}

O percentual de gordura (\%G) foi estimado pelo método duplamente indireto, mensurando-se as dobras cutâneas tricipital e subescapular. Utilizou-se o protocolo de Slaughter et al..$^{25}$ para executar tal estimativa. Essas medidas foram realizadas de forma rotacional e coletadas três vezes, considerando-se a média dos valores.

A massa corporal foi mensurada por uma balança digital portátil da marca Tanita, com precisão de $100 \mathrm{~g}$ e capaci- dade máxima de $200 \mathrm{~kg}$. Utilizou-se estadiômetro portátil, com precisão de 0,1 cm e altura máxima de 2,20 m, da marca Welmy, para mensurar a estatura das atletas. Calculou-se o índice de massa corporal (IMC) a partir dessas medidas [IMC $=$ massa corporal $(\mathrm{kg}) /$ estatura $\left.\left(\mathrm{m}^{2}\right)\right]$.

\section{Nível econômico}

Foi obtido mediante aplicação do "Critério de Classificação Econômica Brasil" desenvolvido pela Associação Brasileira de Empresas de Pesquisa (ABEP). O Critério de Classificação Econômica Brasil enfatiza sua função de estimar o poder de compra das pessoas e famílias urbanas, abandonando a pretensão de classificar a população em termos de "classes sociais". Esse instrumento avalia a quantidade de itens de conforto (automóvel, geladeira, televisão etc.) adquiridos, além de identificar o grau de instrução do chefe de família. Ele remete a pontuações variando de 0 a 34, ou seja, quanto maior o escore, maior é o nível econômico.

\section{Dados demográficos}

Aplicou-se um questionário qualitativo a fim de avaliar dados demográficos como: idade, etnia (branco, negro ou amarelo), nível competitivo (regional, estadual ou nacional) e horas de treino por dia.

\section{Procedimentos}

A coleta de dados ocorreu durante os meses de março, abril e maio de 2011 em salas disponibilizadas pelos clubes participantes da pesquisa. Uma semana antes da aplicação dos questionários e aferições antropométricas, foram distribuídos os Termos de Consentimento Livre e Esclarecido (TCLE), para que os pais das esportistas autorizassem a coleta de dados. Na semana seguinte, os pesquisadores aplicaram os instrumentos e realizaram aferições antropométricas nas atletas que apresentaram o TCLE assinado pelo responsável.

Após breve explicação sobre a pesquisa, as jovens responderam aos questionários individualmente. O tempo médio de preenchimento foi de 35 minutos. As medidas antropométricas (peso, estatura e dobras cutâneas) foram aferidas logo após esse primeiro momento.

\section{Análise estatística}

Utilizaram-se média, desvio-padrão e frequências para descrever as variáveis do estudo. A estatística inferencial foi aplicada para relatar a consistência interna (alfa de Cronbach) dos questionários. Conduziu-se regressão linear múltipla stepwise para averiguar a influência que as variáveis do estudo (insatisfação corporal, comprometimento psicológico ao exercício, antropometria, nível econômico, nível competitivo e etnia) exerciam sobre o comportamento alimentar em jovens atletas femininas. O tratamento dos dados foi realizado no software SPSS 17.0, adotando-se nível de significância de $5 \%$. 


\section{RESULTADOS}

Tabela 1. Média e desvio-padrão de idade, IMC, \%G, CES, BSQ e CCEB de jovens atletas femininas (Juiz de Fora/MG, 2011)

\begin{tabular}{lc}
\hline Variável & Média (desvio-padrão) \\
\hline EAT-26 & $11,89( \pm 7,92)$ \\
Idade & $14,52( \pm 2,00)$ \\
IMC & $20,43( \pm 3,17)$ \\
$\% G$ & $23,03( \pm 6,22)$ \\
CES & $676,90( \pm 219,17)$ \\
BSQ & $75,45( \pm 28,81)$ \\
CCEB & $21,25( \pm 4,70)$ \\
\hline
\end{tabular}

IMC: índice de massa corporal; \%G: percentual de gordura; CES: Commitment Exercise Scale; BSQ: Body Shape Questionnaire; CCEB: Critério de Classificação Econômica Brasil.

A tabela 2 apresenta a distribuição de frequência da etnia e do nível competitivo da amostra do presente estudo. Nota-se que grande parte dessas jovens era branca $(80,2 \%)$ e competia em âmbito nacional (60,3\%).

Tabela 2. Distribuição de frequência das classificações de etnia e nível competitivo (NC) em jovens atletas femininas (Juiz de Fora/ MG, 2011)

\begin{tabular}{llcc}
\hline Variável & Classificação & Frequência $(\mathbf{n})$ & Frequência $(\%)$ \\
\hline Etnia & Branca & 93 & 80,2 \\
& Negra & 19 & 16,4 \\
& Amarela & 4 & 3,4 \\
NC & Regional & 24 & 20,7 \\
& Estadual & 22 & 19,0 \\
& Nacional & 70 & 60,3 \\
\hline
\end{tabular}

NC: nível competitivo.

Em relação à influência das variáveis da pesquisa sobre o comportamento alimentar, alguns achados merecem destaque. Somente o percentual de gordura $(\% G)(p<0,05)$ e a insatisfação corporal (BSQ) $(p<0,01)$ modularam de forma significativa o comportamento alimentar em jovens atletas femininas. Ademais, o modelo de regressão múltipla explicou em 30\% a variância do comportamento alimentar inadequado nessas adolescentes.

Tabela 3. Regressão linear múltipla utilizando IMC, \%G, CES, BSQ, CCEB, etnia e NC como variáveis explicativas sobre a variância do comportamento alimentar em jovens atletas femininas (Juiz de Fora/MG, 2011)

\begin{tabular}{lcccccc}
\hline Variável & Bloco & $\mathbf{B}$ & $\mathbf{R}$ & $\mathbf{R}^{2}$ & $\mathbf{R}^{2 *}$ & $p$ valor \\
\hline IMC & 1 & 0,05 & 0,15 & 0,02 & 0,02 & $p \leq 0,09$ \\
\%G & 1 & 0,14 & 0,18 & 0,03 & 0,02 & $p \leq 0,05$ \\
CES & 2 & 0,06 & 0,18 & 0,03 & 0,02 & $p \leq 0,09$ \\
BSQ & 3 & 0,16 & 0,63 & 0,40 & 0,39 & $p \leq 0,01$ \\
CCEB & 4 & 0,09 & 0,11 & 0,01 & 0,004 & $p \leq 0,24$ \\
Etnia & 4 & 0,15 & 0,04 & 0,02 & 0,007 & $p \leq 0,66$ \\
NC & 4 & 0,19 & 0,12 & 0,01 & 0,005 & $p \leq 0,21$ \\
Todos & & 8,51 & 0,55 & 0,30 & 0,24 & $p \leq 0,01$ \\
\hline
\end{tabular}

$\mathrm{R}^{2 *}: \mathrm{R}^{2}$ ajustado; IMC: índice de massa corporal; \%G: percentual de gordura; CES: Commitment Exercise Scale; BSQ: Body Shape Questionnaire; CCEB: Critério de Classificação Econômica Brasil; NC: nível competitivo.

\section{DISCUSSÃO}

O presente estudo teve como premissa analisar a influência de fatores de risco sobre o comportamento alimentar em jovens atletas femininas. Os resultados evidenciaram que a insatisfação corporal e o percentual de gordura foram as únicas variáveis que modularam as pontuações do EAT-26. Contudo, o modelo de regressão linear múltipla explicou 1/3 da variância do comportamento alimentar nessas esportistas.

A insatisfação corporal, por si só, influenciou 40\% do comportamento alimentar das atletas da presente pesquisa. Parece que no público feminino a insatisfação com o corpo é a principal variável explicativa para a alimentação inadequada, tanto em escolares ${ }^{12,13}$ quanto em atletas ${ }^{10,21}$. Talvez as atletas criem hábitos alimentares considerados patológicos como vômitos autoinduzidos, restrição alimentar, uso de laxativos e diuréticos almejando otimizar o desempenho esportivo ${ }^{5,10}$, pois elas costumam associar a magreza ao rendimento atlético ${ }^{17}$. Nesse sentido, De Bruin et al. ${ }^{11}$ ressaltam que esportistas do sexo feminino, quando almejam maximizar seu rendimento competitivo, geralmente direcionam suas atitudes para a perda de peso corporal. Sendo assim, meninas atletas podem pressupor que o segredo para melhorar o pico de desempenho esportivo seja atingir morfologia corporal com baixo percentual de gordura.

Os achados do presente estudo em relação ao comprometimento psicológico ao exercício mostraram que este não influenciou significativamente os escores do EAT-26. Entretanto, Modolo et al. ${ }^{18}$ salientam que a dependência de exercício físico ou, simplesmente, o excesso de atividade física podem ser considerados fatores de risco para a alimentação inadequada em atletas. Parece que em jovens esportistas femininas essa hipótese não procede, o que pode ser identificado nos resultados do presente manuscrito. Talvez o comprometimento psicológico ao exercício na presente amostra tenha sido baixo, pois grande parte dessas jovens participava de competições de nível nacional, o que pode ser considerado como fator protetor contra instalação da dependência de exercício físico, visto que autores afirmam que essa dependência é mais encontrada em atletas que competem em níveis inferiores ${ }^{7,18}$.

Remetendo-se às variáveis antropométricas, identificou-se apenas influência significativa do percentual de gordura sobre o comportamento alimentar. No entanto, o percentual de gordura explicou somente 3\% da variância do EAT-26. Parece que características morfológicas não exercem grande influência nos hábitos alimentares de atletas femininas. Segundo Monthuy-Blanc et al. ${ }^{26}$, o surgimento do comportamento alimentar de risco para TA em jovens atletas independe dos valores de peso, IMC e gordura corporal. Talvez as atletas sintam-se mais pressionadas pelos treinadores, familiares e patrocinadores em atingir uma estética magra do que por suas características corporais, podendo refletir 
na aquisição de comportamentos alimentares deletérios à saúde ${ }^{7,9}$.

O presente estudo não encontrou influência significativa da etnia e do nível econômico sobre o comportamento alimentar inadequado de atletas femininas. Entretanto, pesquisadores afirmam que os hábitos alimentares não saudáveis estão presentes nos estratos mais altos da sociedade e em indivíduos de etnia branca ${ }^{27}$. No entanto, exalta-se que esses estudos foram desenvolvidos com escolares. Portanto, acredita-se que esta seja a primeira pesquisa a envolver a etnia e o nível econômico como variáveis explicativas do comportamento alimentar na população de esportistas competitivos, o que dificulta comparações e generalizações.

Em relação ao nível competitivo, também não se encontrou influência significativa dele sobre as pontuações do EAT-26. Parece que a participação em competições não modula hábitos alimentares inadequados em atletas femininas. Em contrapartida, autores afirmam que o nível de competitividade elevado é considerado fator de risco para a alimentação inadequada ${ }^{7,21}$, podendo levar a atleta a desenvolver algum tipo de transtorno da alimentação. Entretanto, ressalta-se que ainda não existe nenhum indicador de nível competitivo que seja confiável. Pesquisas têm utilizado a participação mais importante em competição, número de medalhas adquiridas em torneios ou quantidade de horas de treino semanais como tais indicadores ${ }^{2,7,21}$.

Finalmente, observa-se que 30\% do comportamento alimentar inadequado de jovens atletas femininas foi modulado pela interação de todas as variáveis no modelo de regressão linear múltipla stepwise. Apesar de encontrar-se significância estatística no modelo, estimava-se que a variância do comportamento alimentar em esportistas femininas fosse mais bem explicada a partir dessas variáveis. Parece que os hábitos alimentares não saudáveis no público esportista feminino são extremamente complexos, necessitando ser mais bem explorados.

O presente estudo apresentou limitações que merecem ser apontadas. Pesquisadores afirmam que os participantes podem não responder a questionários autoaplicáveis com fidedignidade, ainda mais se tratando de atletas, que podem associar sua participação à permanência na equipe. Portanto, é comum que atletas, mesmo com problemas psicológicos, mascarem suas síndromes omitindo-as por meio de respostas não confiáveis a partir de instrumentos desse tipo. Além disso, a presente pesquisa utilizou instrumentos que não são validados na literatura científica para avaliar etnia e níveis econômicos e competitivos. No entanto, ressalta-se que vários estudos têm utilizado os mesmos métodos para avaliar essas variáveis ${ }^{7,27}$. Por fim, salienta-se que este estudo apresentou resultados interessantes que merecem ser discutidos na literatura.

Sugere-se que sejam realizadas mais pesquisas no âmbito esportivo competitivo, utilizando o sexo masculino como amostra e englobando mais variáveis de cunho psicológico que possam melhor explicar variáveis comportamentais. Ademais, sugerem-se também estudos que incluam outras modalidades esportivas e averigue se o tipo de esporte exerce influência sobre o comportamento alimentar de risco para TA.

\section{CONCLUSÃO}

Os resultados do presente estudo permitem concluir que o comportamento alimentar inadequado de jovens atletas femininas foi influenciado basicamente pela insatisfação corporal. No entanto, são necessários mais estudos com esse público para esclarecer possíveis fatores de risco sobre hábitos alimentares não saudáveis.

Programas de avaliação e orientação, tanto psicológica quanto nutricional, são necessários no âmbito esportivo. Essas iniciativas devem ser desenvolvidas com o caráter de prevenção e detecção de doenças como os TA em atletas.

\section{AGRADECIMENTOS}

Às atletas participantes deste estudo, pela colaboração; ao Laboratório de Estudos do Corpo (UFJF), pela disponibilização dos instrumentais do estudo; e ao Conselho Nacional de Desenvolvimento Científico e Tecnológico (CNPq), pelo financiamento do projeto.

\section{REFERÊNCIAS}

1. Gomes JP, Legnani E, Legnani RFS, Gregório NP, Souza RK. Associação entre comportamento alimentar, consumo de cigarro, drogas e episódios depressivos em adolescente. Rev Nutr. 2010;23(5):755-62.

2. Schaal K, Tafflet M, Nassif H, Thibault V, Pichard C, Alcotte M, et al. Psychological balance in high level athletes: gender-based differences and sport-specific patterns. PLoS One. 2011;6(5)::19007.

3. Kirsten VR, Fratton F, Porta NBD. Transtornos alimentares em alunas de nutrição do Rio Grande do Sul. Rev Nutr. 2009;22(2):219-27.

4. Sundgot-Borgen J, Torstveit MK. Prevalence of disorders in elite athletes in higher than in the general population. Clin J Sport Med. 2004;14(1):25-32.

5. Perini TA, Vieira RS, Vigário OS, Oliveira GL, Ornellas JS, Oliveira FP. Transtorno do comportamento alimentar em atletas de elite de nado sincronizado. Rev Bras Med Esporte. 2009;15(1):54-7.

6. Artioli GG, Scagliusi FB, Polacow V0, Gualano B, Lancha Junior AH. Magnitude e métodos de perda rápida de peso em judocas de elite. Rev Nutr. 2007;20(3):307-15.

7. Denoma JMH, Scaringi V, Gordon KH, Van Orden KA, Joiner TE. Eating disorder symptoms among undergraduate varsity athletes, club athletes, independent exercisers. and nonoexercises. Int J Eat Disord. 2009;12(1):47-53.

8. Vieira JLL, Amorim HZ, Vieira LF, Amorim AC, Rocha PGM. Distúrbios de atitudes alimentares e distorção da imagem corporal no contexto competitivo da ginástica rítmica. Rev Bras Med Esporte. 2009;15(6):410-4

9. Gomes R, Silva L. Desordens alimentares e perfeccionismo: um estudo com atletas portugueses. Psicologia Rev. 2010;16(3):469-89. 
10. Krentz EM, Warschburger P. Sports-related correlates of disordered eating in aesthetic sports. Psychol Sport Exerc. 2011;44(3):315-21.

11. De Bruin AP, Oudejans RRD, Bakker FC. Dieting and body image in aesthetic sports: a comparison of Dutch female gymnasts and non-aesthetic sport participants. Psychol Sport Exerc. 2007;8(4):507-20

12. Conti MA, Frutuoso MFP, Gambardella AMD. Excesso de peso e insatisfação corporal em adolescentes. Rev Nutr. 2005;18(4):491-7.

13. Martins CR, Pelegrini A, Matheus SC, Petroski EL. Insatisfação com a imagem corporal e a relação com estado nutricional, adiposidade corporal e sintomas de anorexia e bulimia em adolescentes. Rev Psiquiatr Rio Gd Sul. 2010;32(1):19-23.

14. Scherer FC, Martins CB, Pelegrini A, Matheus SC, Petroski EL. Imagem corporal em adolescentes: associação com a maturação sexual e sintomas de transtornos alimentares. Rev Bras Psiquiatr. 2010;59(3):198-202.

15. Alves E, Vasconcelos FAG, Calvo MCM, Neves J. Prevalência de sintomas de anorexia nervosa e insatisfação com a imagem corporal em adolescentes do sexo feminino do Município de Florianópolis, Santa Catarina, Brasil. Cad Saude Publica. 2008;24(3):503-12.

16. Castro IRR, Levy RB, Cardoso LO, Passos MD, Sardinha LMV, Tavares LF, et al. Imagem corporal, estado nutricional e comportamento com relação ao peso entre adolescentes brasileiros. Ciênc Saúde Coletiva. 2010;15(Supl 2):3099-108.

17. Torstveit MK, I Sundgot-Borgen. The female athlete triad exists in both elite athletes and controls. Med Sci Sports Exerc. 2005;37(1):1449-59.

18. Modolo VB, Antunes HKM, Gimenez PRB, Santiago MLM, Tufik S, Mello MT. Negative addiction to exercise - are there differences between genders?. Clinics. 2011;66(2):255-60.
19. Dunker KLL, Fernandes CPB, Filho DC. Influência do nível socioeconômico sobre comportamentos de risco para transtornos alimentares em adolescentes. J Bras Psiquiatr. 2009;58(3):156-61.

20. Sampei MA, Singulem DM, Novo NF, Juliano Y, Colugnati, FA. Atitudes alimentares e imagem corporal em meninas adolescentes de ascendência nipônica e caucasiana em São Paulo (SP). J Pediatria. 2009;85(2):122-8.

21. Haase AM. Weight perception in female athletes: associations with disordered eating correlates and behavior. Eat Behav. 2011;12(1):64-7.

22. Bighetti F, Santos (B, Santos JE, Ribeiro RPP. Tradução e avaliação do Eating Attitudes Test em adolescentes do sexo feminino de Ribeirão Preto, São Paulo. J Bras Psiquatr. 2004;53(6):339-46.

23. Conti MA, Cordás TA, Latorre MRDO. Estudo de validade e confiabilidade da versão brasileira do Body Shape Questionnaire (BSQ) para adolescentes. Rev Bras Saúde Mater Infant. 2009;9(3):331-8

24. Teixeira PC, Hearst N, Matsudo SMM, Cordás TA, Conti MA. Adaptação transcultural: tradução e validação de conteúdo da versão brasileira do Commitment Exercise Scale. Rev Psiq Clin. 2011;38(1):24-8.

25. Slaughter MH, Lohman TG, Boileau R, Hoswill CA, Stillman RJ, Yanloan MD. Skinfold equations for estimation of body fatness in children and youth. Hum Biol. 1988;60(3):709-23.

26. Monthuy-Blanc J, Maiano C, Therme P. Prevalence of eating disorders symptoms in nonelite ballet dancers and basketball players: an exploratory and controlled study among French adolescent girls. Rev Epidemiol Sante Publique. 2010;1(2):1-10.

27. Sampei MA, Singulem DM, Novo NF, Juliano Y, Colugnati FA. Atitudes alimentares e imagem corporal em meninas adolescentes de ascendência nipônica e caucasiana em São Paulo (SP). J Pediatria. 2009;85(2):122-8. 